\section{European \\ Neurology}

Eur Neurol 2001;45:1-2

\title{
Message from the Editor
}

\author{
J. Bogousslavsky, Lausanne
}

It is the policy of the journal to rotate the members of the Editorial Board every now and then and I would like to thank the following people for their great efforts over the past years:

\section{A. Albanese, Milan, Italy}

T.D. Bird, Seattle, Wash., USA

M. Fisher, Worcester, Mass., USA

F.G.A. van der Meché, Rotterdam, The Netherlands

I would also like to welcome the new members of the Editorial Board and thank them for agreeing to support European Neurology:

C. Bassetti, Bern, Switzerland

D. Dressler, Rostock, Germany

P.J. Goadsby, London, UK

W. Poewe, Innsbruck, Austria

The following names are those of ad hoc reviewers, to whom I would also like to express my gratitude (October 1999 - October 2000):

A. Aguzzi, Zürich, Switzerland

A. Albanese, Milan, Italy

G.M. Annoni, Genève, Switzerland

Z. Argov, Jerusalem, Israel

C. Bassetti, Bern, Switzerland

R.W. Baumgartner, Zürich, Switzerland

S.R. Benbadis, Tampa, Fla., USA

J. Berciano, Santander, Spain

A. Bernasconi, Montréal, Que., Canada
A. Berney, Lausanne, Switzerland

T.D. Bird, Seattle, Wash., USA

W.T. Blume, London, Ont., Canada

N. Bornstein, Tel Aviv, Israel

F.-X. Borruat, Lausanne, Switzerland

A. Brice, Paris, France

P. Burckhardt, Genève, Switzerland

J.M. Burgunder, Bern, Switzerland

E. Busch, Essen, Germany

L.R. Caplan, Boston, Mass., USA

A. Carota, Lausanne, Switzerland

A. Carruzzo, Lausanne, Switzerland

P. Cesaro, Créteil, France

H. Chabriat, Paris, France

S. Clarke, Lausanne, Switzerland

G. Comi, Milan, Italy

C. Confavreux, Lyon, France

A. Culebras, Syracuse, N.Y., USA

A.R. Damasio, Iowa City, Iowa, USA

S. Di Donato, Milan, Italy

H.-C. Diener, Essen, Germany

M. Dieterich, München, Germany

M.A. Duchosal, Lausanne, Switzerland

A. Engel, Rochester, Minn., USA

A. Filla, Naples, Italy

M. Fisher, Worcester, Mass., USA

P. Francioli, Lausanne, Switzerland

N. Futrell, Murray, Utah, USA

\section{KARGER}

๑) 2001 S. Karger AG, Basel

Fax +4161306 1234

E-Mail karger@karger.ch

www.karger.com
Accessible online at: www. karger.com/journals/ene 
J. Ghika, Lausanne, Switzerland

J. van Gijn, Utrecht, The Netherlands

F. Graus, Barcelona, Spain

S. Greenberg, Boston, Mass., USA

J.H. Growdon, Boston, Mass., USA

W. Hacke, Heidelberg, Germany

J.J. Hauw, Paris, France

H. Hénon, Lille, France

J. Hildebrand, Brussels, Belgium

L. Hirt, Lausanne, Switzerland

C. Hock, Zürich, Switzerland

D. Inzitari, Florence, Italy

P. Jallon, Genève, Switzerland

M. Kawamura, Tokyo, Japan

J. Kesselring, Valens, Switzerland

J. Kimura, Iowa City, Iowa, USA

T. Kuntzer, Lausanne, Switzerland

T. Landis, Genève, Switzerland

K.L. Leenders, Groningen, The Netherlands

N. Leigh, London, UK

G.L. Lenzi, Rome, Italy

P. Leuenberger, Lausanne, Switzerland

D. Leys, Lille, France

J.M. Léger, Paris, France

P. Low, Rochester, Minn., USA

H.P. Ludin, St. Gallen, Switzerland

E. Lugaresi, Bologna, Italy

P. Maeder, Lausanne, Switzerland

P.J. Magistretti, Lausanne, Switzerland

C. Masson, Clichy, France

C. Mathias, London, UK

H. Mattle, Bern, Switzerland

O. Meienberg, Binningen, Switzerland

R.A. Meuli, Lausanne, Switzerland

D. Mojon, St. Gallen, Switzerland

V. Mooser, Lausanne, Switzerland

H.W. Moser, Baltimore, Md., USA

T. Munsat, Boston, Mass., USA
B. Nater, Lausanne, Switzerland

H.-W. Pfister, München, Germany

C. Pierrot-Deseilligny, Paris, France

P. Pollak, Grenoble, France

O. Rascol, Toulouse, France

J. de Reuck, Gent, Belgium

W.A. Rocca, Rochester, Minn., USA

L.P. Rowland, New York, N.Y., USA

G. Said, Le Kremlin-Bicêtre, France

J.W.A.S. Sander, Gerrards Cross, UK

P. Scheltens, Amsterdam, The Netherlands

M. Schluep, Lausanne, Switzerland

M. Seeck, Genève, Switzerland

V. Silani, Milan, Italy

C.J.M. Sindic, Bruxelles, Belgium

D.O. Slosman, Genève, Switzerland

R. Stajzel, Genève, Switzerland

F. Staub, Lausanne, Switzerland

A.J. Steck, Basel, Switzerland

M. Sturzenegger, Bern, Switzerland

N. de Tribolet, Lausanne, Switzerland

J.J. Van Hilten, Leiden, The Netherlands

L. Vercueil, Grenoble, France

M. Vidailhet, Paris, France

J.G. Villemure, Lausanne, Switzerland

A. Vincent, Oxford, UK

F.J.G. Vingerhoets, Lausanne, Switzerland

J.-P.G. Vonsattel, Charlestown, Mass., USA

P. Vuadens, Sion, Switzerland

B. Waeber, Lausanne, Switzerland

G.K. Wenning, Innsbruck, Austria

H.G. Wieser, Zürich, Switzerland

J.H.J. Wokke, Utrecht, The Netherlands

A. Yamadori, Sendai, Japan

D. Zegers de Beyl, Brussels, Belgium 